\title{
Simulation du bouchon vaseux dans l'estuaire de la Seine : tentative de hiérarchisation des processus de formation
}

\author{
Isabelle Brenon et Pierre Le Hir \\ Direction de l'Environnement et de l'Aménagement Littoral \\ IFREMER/Centre de BREST - B.P. 70 - 29280 PLOUZANE
}

\begin{abstract}
A depth-averaged and a three-dimensional models for tide propagation and fine sediment transport are used in order to determine the respective role of several processes on the turbidity maximum behaviour in the Seine estuary. Without any gravitational circulation, an accumulation of particles is reproduced during the first spring tides at a location in agreement with field measurements. This result confirms the dominant role of tidal pumping. The bathymetry influences the location of deposited sediment patterns and, consequently, modifies the location of the turbidity maximum. The sediment behaviour (settling. erodability) influences the concentration and the location of all these tidal-induced sediment. patterns. On the other hand, the salinity gradients induce a density circulation, which helps to maintain particles in the estuary (concentrations are thus higher) and modifies the extension of the turbidity maximum.
\end{abstract}

\section{Introduction}

Une accumulation de particules en suspension, appelée "bouchon vaseux", se produit souvent dans les estuaires. Les facteurs principalement reconnus comme influençant la dynamique de ce bouchon vaseux sont le "pompage tidal" qui résulte de l'asymétrie de la propagation de la marée (Allen et al, 1980) et la circulation de densité (Postma, 1967) qui s'établit dans la zone de gradient de densité entre cau douce et eau de mer. Dans le cas de l'estuaire de la Seine, objet du programme scientifique pluridisciplinaire Seine Aval, les effets des piégeages (le long des côtes) dans les secteurs de faible hydrodynamisme, du comportement du matériel (capacité d'érosion, de dépôt, de floculation ....) et des remises en suspension par les vagues sembient moduler les facteurs précédents. Seule la modelisation permet de détemininer le rôle de chacun de ces processus sur le comportement des structures turbides.

L'estuaire de la Seine est un estuaire macrotidal ( $7 \mathrm{~m}$ de marnage) avec un débit moyen d'environ $500 \mathrm{~m}^{3} / \mathrm{s}$. Il est constitué du chenal de navigation du Port Autonome de Rouen (endigué jusqu'à la sortie de l'estuaire) et, de part et d'autre à son embouchure, de zones peu profondes en voie de comblement par des sables au sud et des vases au nord (Avoine et al, 1996). Le bouchon vaseux est principalement localisé dans le chenal de navigation entre Honfleur et Tancarville.

Afin de hiérarchiser les mécanismes affectant le comportement du bouchon vaseux dans l'estuaire de la Seine, deux modèles de transport des sédiments fins 
ont été développés. En raison du fort marnage dans cet estuaire, on peut penser que l'asymétrie de la propagation de la marée est prédominante, ce qui justifie l'utilisation initiale d'un modèle bidimensionnel horizontal ne rendant pas compte des gradients de salinité. Le modèle tridimensionnel permet, quant à lui, de considérer tous les forçages. Dans cette étude, seuls les sédiments fins (définis par leurs propriétés cohésives et transportés exclusivement en suspension) sont pris en compte, ce qui se justifie par leur rôle prépondérant dans la constitution du bouchon vaseux, par l'extension en cours des bancs de vases à l'embouchure (Avoine et al, 1996), par leur capacité à être remis en suspension et à adsorber les contaminants.

\section{Présentation des modèles}

\subsection{Le modèle bidimensionnel horizontal}

Pour calculer les hauteurs d'eau et les courants, les équations de la mécanique des fiuides, intégrées sur la verticale après hypothèse d'hydrostaticité et approximation de Boussinesq (système de Saint-Venant), sount Iésolues selon une méthode aüx différences finies implicite en directions alternées. Le modèle bidimensionnel horizontal s'étend de la partie est de la Baie de Seine à la première écluse (Poses en amont de rouen). Il utilise une bathymétrie précise et récente (Port Autonome de Rouen, 1993) à l'embouchure et dans le secteur de bouchon vaseux. Pour réduire ies temps de calcul tout en gardant une grande résolution dans la zone d'étude, un maillage rectiligne variable (mailles de 200 à $4000 \mathrm{~m}$ ) a été utilisé (figure 1). Le modèle tient compte des bancs découvrants de façon automatique.

A l'aval, les conditions aux limites en hauteur d'eau ont été introduites sous la forme d'une marée réelle calculée à l'aide de la composition harmonique des 26 ondes principales de marée (mesures du Service Hydraulique et Océanographique de la Marine et modèle réduit toumant du laboratoire Coriolis de l'Université de Grenoble). A l'amont, un débit quotidien d'eau douce est introduit. La propagation de la marée est reproduite (en particulier, la double pleine mer, caractéristique à l'embouchure) et validée. L'asymétrie des courants et des étales, accentuée lors de la propagation de l'onde vers l'amont, est simulée (Brenon et Le Hir, 1996a).

Le modèle résout, pour le calcul de la concentration, l'équation de conservation de la masse qui rend compte des phénomènes d'advection, de dispersion et des échanges avec le fond sédimentaire. Le dépôt est écrit selon la formulation de Krone (1962), et l'érosion selon celle de Partheniades (1965). Le schéma de résolution explicite est écrit en différences finies selon l'algorithme de Bott (1989). En chaque point de la grille du modèle 2DH, un modèle de consolidation unidimensionnel vertical multi-couches rend compte du tassement, en supposant que la vitesse de sédimentation ne dépend que de la concentration locale. Il résout une équation de conservation de la masse de sédiments entre des niveaux fixes, suivant un schéma semi-implicite en différences finies. La tension critique 
d'érosion est donnée sous la forme d'une expression empirique, en fonction de la concentration du sédiment $\left(\tau_{c e}=0.005 \mathrm{C}\right)$. La discrétisation verticale est de l'ordre de $2 \mathrm{~cm}$. Le modèle calcule la hauteur de sédiment et le profil de densité dans la vase. Le nombre de couches et l'épaisseur de la couche de surface varient en fonction des processus de dépôt, d'érosion et de consolidation (Brenon et Le Hir, 1996b).

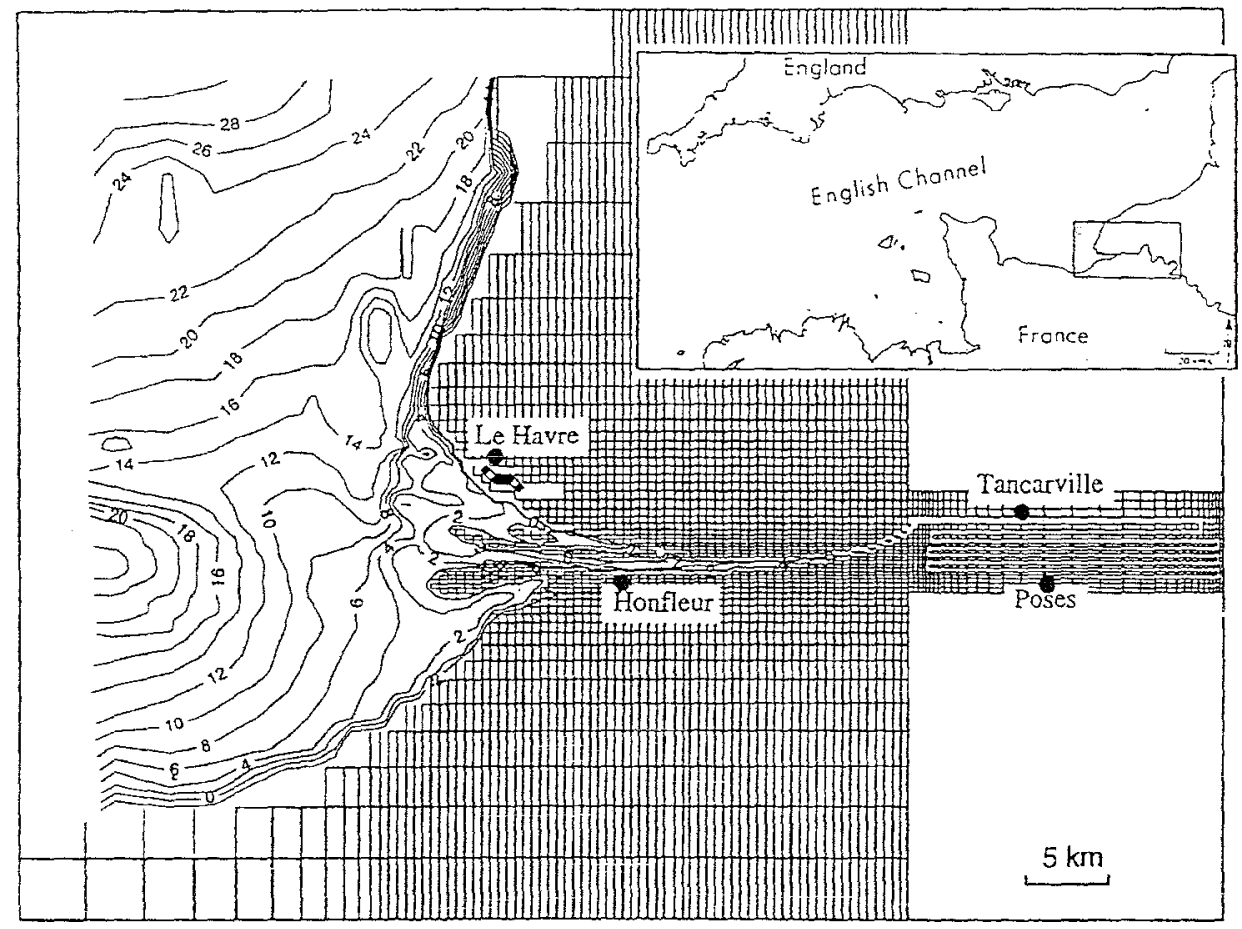

Figure 1 : estuaire de la Seine : localisation, maillage et bathymétrie.

Les processus ont été volontairement simplifiés de façon à montrer le rôle joué par l'hydrodynamique sur les structures turbides. Ainsi, les processus de floculation ne sont pris en compte qu'à travers une vitesse de chute forte $(1 \mathrm{~mm} / \mathrm{s})$, dont l'ordre de grandeur est conforme aux observations in situ les plus récentes (Van Leussen, 1995), en particulier dans l'estuaire de la Seine (Defossez, 1996). La tension de cisaillement critique est choisie forte $\left(\tau_{c d}=10 \mathrm{~N} / \mathrm{m}^{2}\right)$ pour que le dépôt soit toujours possible. Lorsque la turbulence est grande, les dépôts autorisés par le modèle sont instantanément remis en suspension. Une calibration sommaire a ensuite conduit à prendre une valeur de la constante d'érosion égale à $10^{-3} \mathrm{~kg} / \mathrm{m}^{2} / \mathrm{s}$ dans les limites couramment admises dans la littérature $\left(10^{-5}\right.$ à $10^{-2} \mathrm{~kg} / \mathrm{m}^{2} / \mathrm{s}$, in De Nadaillac, 1985). Des tests de sensibilité ont d'ailleurs montré que le modèle est beaucoup moins sensible à ce paramètre qu'à la tension critique d'érosion ou à la vitesse de chute (Brenon, 1997). Ces paramètres seront utilisés en tant que "paramétrage de référence". Le rôle des vagues dans la remise en suspension et les éventuels glissements de vase ne sont pas considérés.

Initialement, il n'y a pas de particules en suspension dans l'estuaire. Afin de montrer l'indépendance de cette structure avec la condition initiale, celle-ci est 
choisie volontairement irréaliste et introduite sous la forme d'un stock de vase facilement érodable et localisé loin du bouchon vaseux. A l'aval, la concentration en sédiment est choisie nulle tandis qu'à l'amont, une charge, proportionnelle au débit fluvial, est introduite.

\subsection{Le modèle tridimensionnel}

Pour calculer hauteurs d'eau et courants, le modèle tridimensionnel (Cugier, 1997) résout les équations de Navier-Stokes en appliquant les hypothèses de Boussinesq et d'hydrostaticité. La résolution de ce système d'équations est basée sur la séparation entre modes barotropes et baroclines. L'élévation de la surface libre et les vitesses moyennes sur la verticale sont calculées par le modèle $2 \mathrm{DH}$. La surface libre est alors fournie au modèle $3 \mathrm{D}$, qui détermine les composantes de la vitesse. Le couplage est assuré par un calcul du frottement dans le modèle 2DH réalisé à partir des vitesses calculées au fond par le modèle 3D, et par la dispersion verticale. L'ensemble est conçu pour être parfaitement conservatif. L'avantage de cette méthode est de permettre un calcul tridimensionnel moins fréquent que le calcul $2 \mathrm{DH}$, contraint par la propagation des ondes de surface. Les coordonnées réelles ont été préférées aux cocrdonnées réduites $(\cdot)$, d'une part pour respecter la forme des structures verticales (telle que celles de la salinité), d'autre part pour éviter les artefacts numériques, qui pourraient apparaitre en raison des forts gradients bathymétriques entre chenal de navigation et bancs de vase latéraux. Dans la direction verticale, les équations sont résolues selon une méthode implicite appliquée à chaque couche de niveaux horizontaux donnés. La fermeture turbulente choisie repose sur le concept de viscosité (et de diffusivité) turbulentes, évaluées selon la théorie de la longueur de mélange. Les effets de stratification sont pris en compte par une réduction de la longueur de mélange en fonction du nombre de Richardson (Brenon, 1997).

Le modèle 3D de transport des sédiments fins est inspiré du modèle $2 \mathrm{DH}$. A chaque pas de temps, les flux horizontaux sont calculés pour "chaque couche" selon l'algorithme de Bott (1989). Puis, l'équation complète de conservation de la masse est résolue implicitement sur la verticale. Dans le calcul $3 \mathrm{D}$, les processus de consolidation ne sont pas pris en compte. Les conditions de simulation sont semblables dans les deux modèles afin de faciliter les comparaisons.

\section{Effet de l'asymétrie de la propagation de la marée}

Les simulations sont faites à débit moyen $\left(500 \mathrm{~m}^{3} / \mathrm{s}\right)$ pendant plusieurs cycles morte eau/vive eau. L'asymétrie de la propagation de la marée tend à favoriser l'érosion des sédiments en flot (et donc leur transport vers l'amont) et provoque un piégeage des particules en aval du secteur où le régime fluvial l'emporte sur la marée. Effectivement, sans gradient de densité, un bouchon vaseux est reproduit en vive eau avec le modèle bidimensionnel horizontal, malgré des formulations sédimentologiques simplifiées (Brenon et Le Hir, 1996b). Ces résultats ont été confirmés par des simulations réalisées avec le modèle tridimensionnel sans prise 

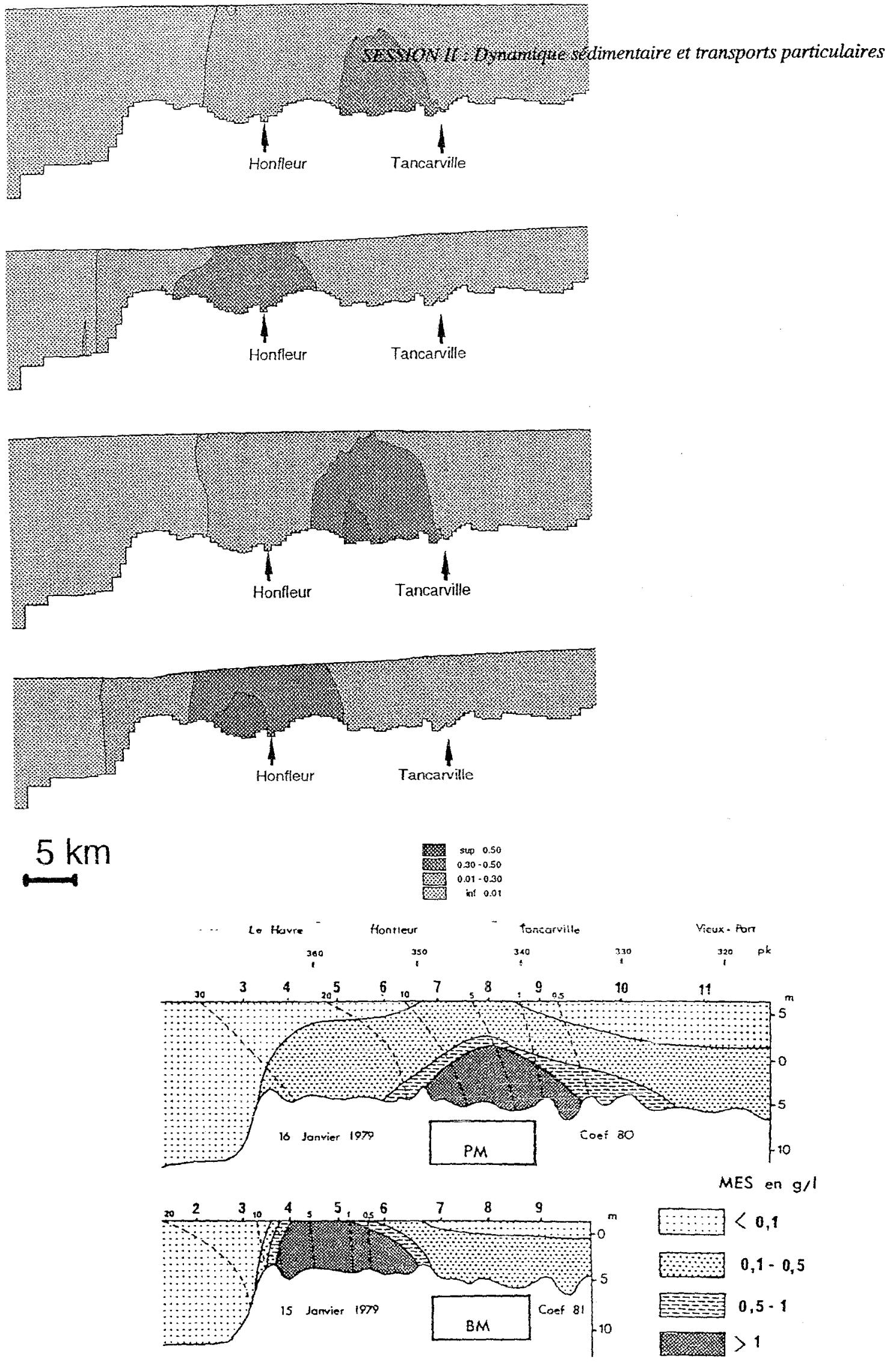

Figure 2 : Distribution des matières en suspension $(g / l)$ en vive eau à pleine mer et à bassc mer pour un débit moyen, calculées avec le modèle tridimensionnel, (a) avec et (b) sans gradients de salinité. Comparaison avec des mesures de la structure verticale du bouchon vaseux (in Avoine, 1981). 
en compte des gradients de densité (figure 2). Par rapport à sa position à débit moyen, le bouchon vaseux est simulé 5 à $10 \mathrm{~km}$ plus en aval en crue et $15 \mathrm{~km}$ plus en amont en étiage. Ce comportement du bouchon vaseux est en accord avec les observations (Brenon, 1997). Ce résultat tend à prouver l'importance du rôle de l'asymétrie de la propagation de la marée sur le comportement du bouchon vaseux dans l'estuaire de la Seine.

\section{Effet des dépôts}

Dans les calculs 2DH, on assiste à un piégeage des sédiments le long des berges en amont de Honfleur, là où les vitesses sont faibles, peut-être favorisé par les irrégularités du maillage "en escalier" (figure 3a). On peut alors se demander si la

(a)
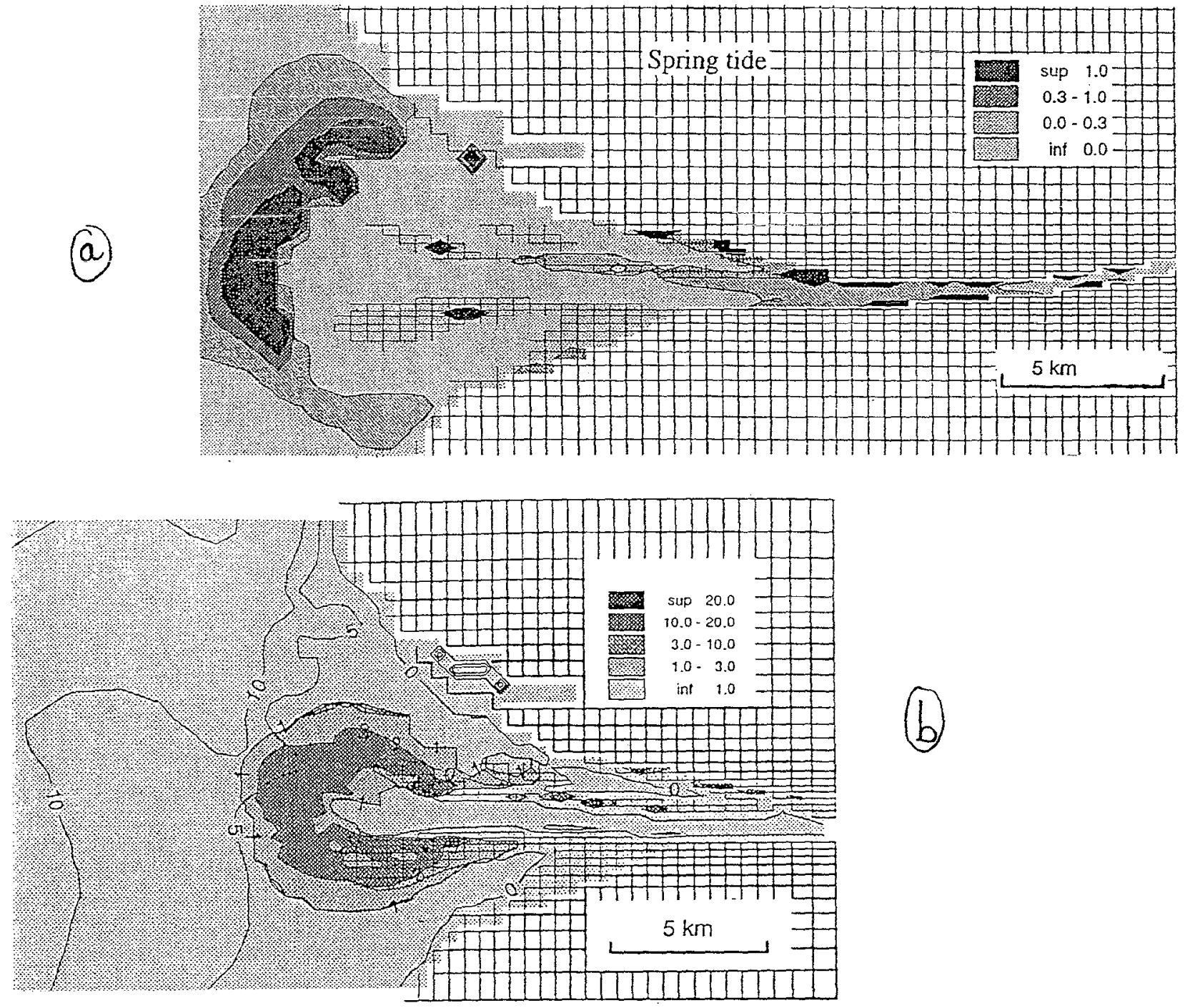

(b)

Figure 3 : localisation des dépôts résiduels $\left(\mathrm{kg} / \mathrm{m}^{2}\right)$ dans l'estuaire de la Seine, après 45 jours de marée réelle

(a) avec le maillage "en escalier", (b) avec le maillage rectifié. 
localisation des MES en vive eau n'est pas forcée par celle de ces dépôts. Les piégeages pourraient maintenir le bouchon vaseux en le "ré-alimentant" en particules en suspension lors de chaque érosion par les courants de revif.

A titre d'essai, le maillage a été rectifié en amont du pont de Normandie. L'effet sur les structures turbides simulées est très marqué : les piégeages entre Honfleur et Tancarville ont disparu en vive eau tandis que les dépôts en aval (figure 3b) ont augmenté (en particulier le long de la digue nord). De ce fait, le bouchon vaseux se déplace vers l'aval. Pour vérifier cet effet, une vasière a été simulée au niveau du Pont de Normandie. Des piégeages de vase sont alors reproduits à ce niveau et le bouchon vaseux "remonte" vers l'amont, ce qui confirme l'interaction importante entre dépôts et MES. Ces simulations ne peuvent être réalisées qu'à titre d'essai, car les conditions réelles ne sont pas connues précisément.

\section{Effet du comportement du matériel}

Afin de déterminer l'influence des paramètres de dépôt et d'érosion sur les structures turbides, une analyse de sensibilité du modèle $2 \mathrm{DH}$ a été réalisée. Le flux d'érosion peut ètre accentué par une augmentation de la constante d'érosion ou par une réduction de la tension de cisaillement critique. Pourtant, la constante d'érosion a une importance moindre sur le comportement des structures sédimentaires, particulièrement quand la tension critique d'érosion est faible (Brenon, 1997), car, dans ce cas, la probabilité d'éroder tout ce qui est érodable est supérieure. Différentes valeurs pour la tension critique d'érosion ont donc été testées. Plus la valeur de ce paramètre augmente, plus le bouchon vaseux est localisé en amont et moins il est concentré (figure 4). En effet, la remise en suspension des particules sédimentaires par les courants de jusant (plus faibles) est alors nettement atténuée et leur transport vers l'aval est réduit. Pour des valeurs

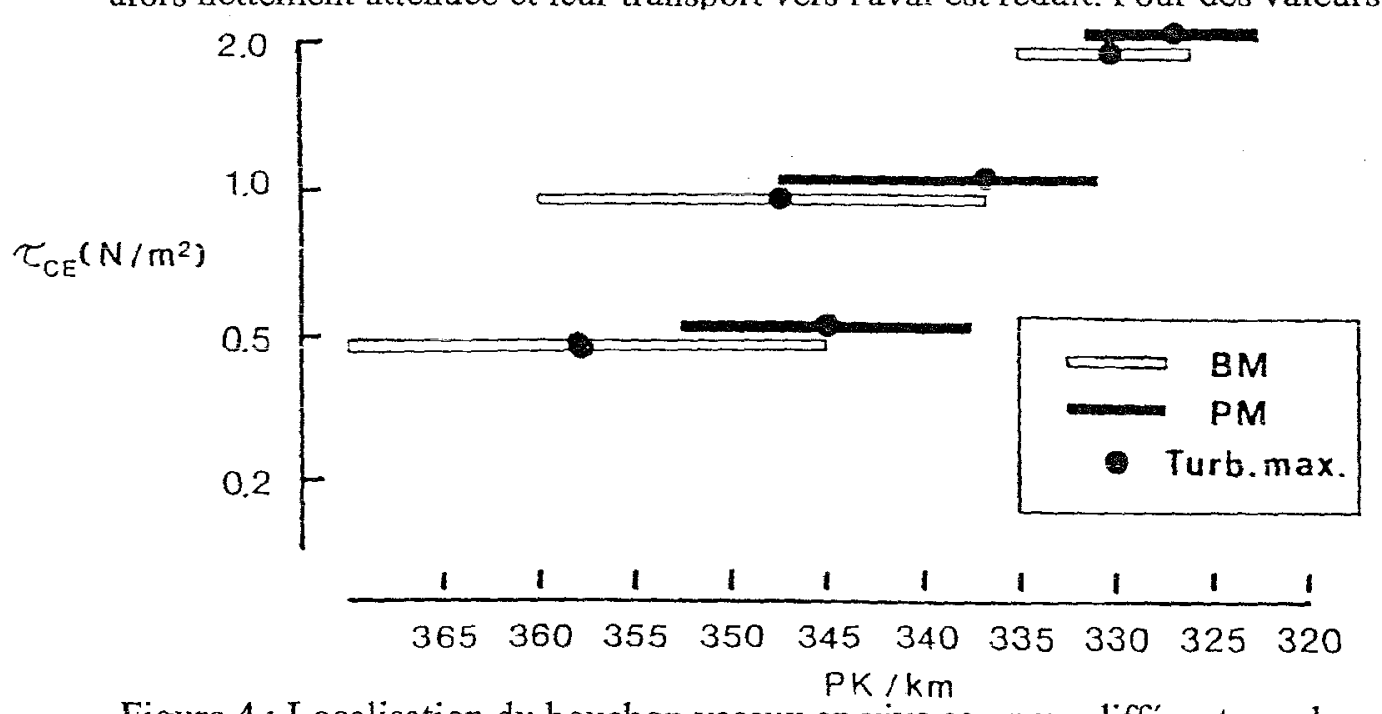

Figure 4 : Localisation du bouchon vaseux en vive eau pour différentes valeurs de la tension critique d'érosion

(la limite de concentration du bouchon vaseux est choisie à $0.3 \mathrm{~g} / \mathrm{l}$ ). 
extrêmes de la tension critique d'érosion, les concentrations sont faibles. En effet, pour des sols peu consolidés $\left(\tau_{c e}=0.2 \mathrm{~N} / \mathrm{m}^{2}\right)$, les particules sont expulsées vers la Baie de Seine, tandis que pour des sols très consolidés $\left(\tau_{\mathrm{ce}}=2 . \mathrm{N} / \mathrm{m}^{2}\right)$, les particules sont difficilement remises en suspension même en vive eau. En conséquence, la tension critique d'érosion, et donc la consolidation, a un rôle important sur le positionnement et la concentration des structures turbides.

Des simulations ont été réalisées pour diverses valeurs, toujours constantes, de la vitesse de chute. Quand la vitesse de chute augmente, dépôts et piégeages sont favorisés et un cisaillement important est nécessaire pour maintenir les particules en suspension. L'effet d'asymétrie entre flot et jusant, et surtout entre les étales de pleine mer et de basse mer, est accentué. En conséquence, le bouchon vaseux se déplace vers l'amont (figure 5). Parallèlement, en raison de la diminution des sections à l'amont, les concentrations augmentent. On note donc que le comportement et la concentration du bouchon vaseux sont très sensibles aux variations de la vitesse de chute (Toorman, 1993). On relève que l'augmentation de la vitesse de chute et de la tension critique d'érosion n'ont pas le même effet sur la concentration des suspensions. En effet, une forte vitesse de chute influence le bouchon vaseux à travers les dépôts aux étales, tandis que la tension critique d'érosion agit directement sur les quantités de sédiments remises en suspension.

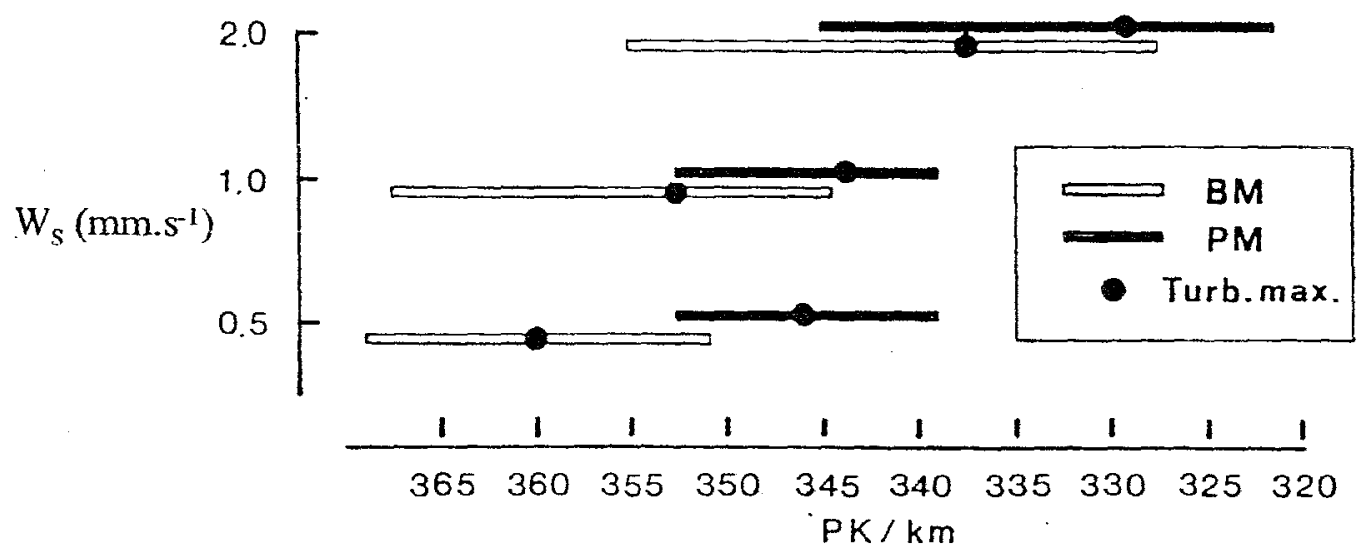

Figure 5 : Localisation du bouchon vaseux en vive eau pour différentes valeurs de la vitesse de chute.

(la limite de concentration du bouchon vaseux est choisie à $0.3 \mathrm{~g} / \mathrm{l}$ ).

\section{Effet des gradients de densité}

Le rôle des gradients de densité est déterminé avec le modèle tridimensionnel en réalisant des simulations avec ou sans ce forçage. Ces gradients tendent à concentrer les matières en suspension (MES) autour du point nodal, situé à la limite amont de la remontée des eaux marines au fond. Ce point nodal ne coincide pas forcément avec la zone d'accumulation liée au "pompage de marée". Un schéma de synthèse de l'influence des gradients de densité sur le comportement des suspensions en vive eau peut être proposé (figure 6). En fonction des 
conditions de débit ou du paramétrage, trois cas sont possibles. Dans le premier, le bouchon vaseux dynamique et la limite des gradients de salinité sont localisés au même endroit, le bouchon vaseux résultant semble alors être "resserré" autour de la position de l'accumulation dynamique. Cette situation est obtenue à débit moyen pour le paramétrage de référence. Dans un deuxième cas, les gradients de salinité sont localisés en aval du bouchon vaseux dynamique, le bouchon vaseux résultant est alors étendu vers l'aval. Cette situation est obtenue quand on augmente la vitesse de chute ou la tension critique d'érosion, mais aussi en crue et en étiage. Dans le troisième cas, les gradients "étirent" le bouchon vaseux dynamique vers l'amont, c'est la situation lors d'une diminution de la vitesse de chute. La localisation de l'accumulation liées aux gradients de densité correspond à la limite de remontée de l'eau de mer et est donc indépendante du comportement du matériel. Le passage de crue à étiage ne fait pas forcément changer de cas, car les gradients de salinité et le bouchon vaseux dynamique se déplacent dans le mème sens (Brenon, 1997).

De plus, des concentrations en MES sont calculées plus faibles dans les simulations sans gradient de densité que dans les simulations où ceux-ci sont pris en compte. Il semble donc qu'un des effets des gradients de densité soit de maintenir les particules dans l'estuaire, ce qui avait aussi été montré dans I'estuaire de la Loire (Le Hir, 1997).

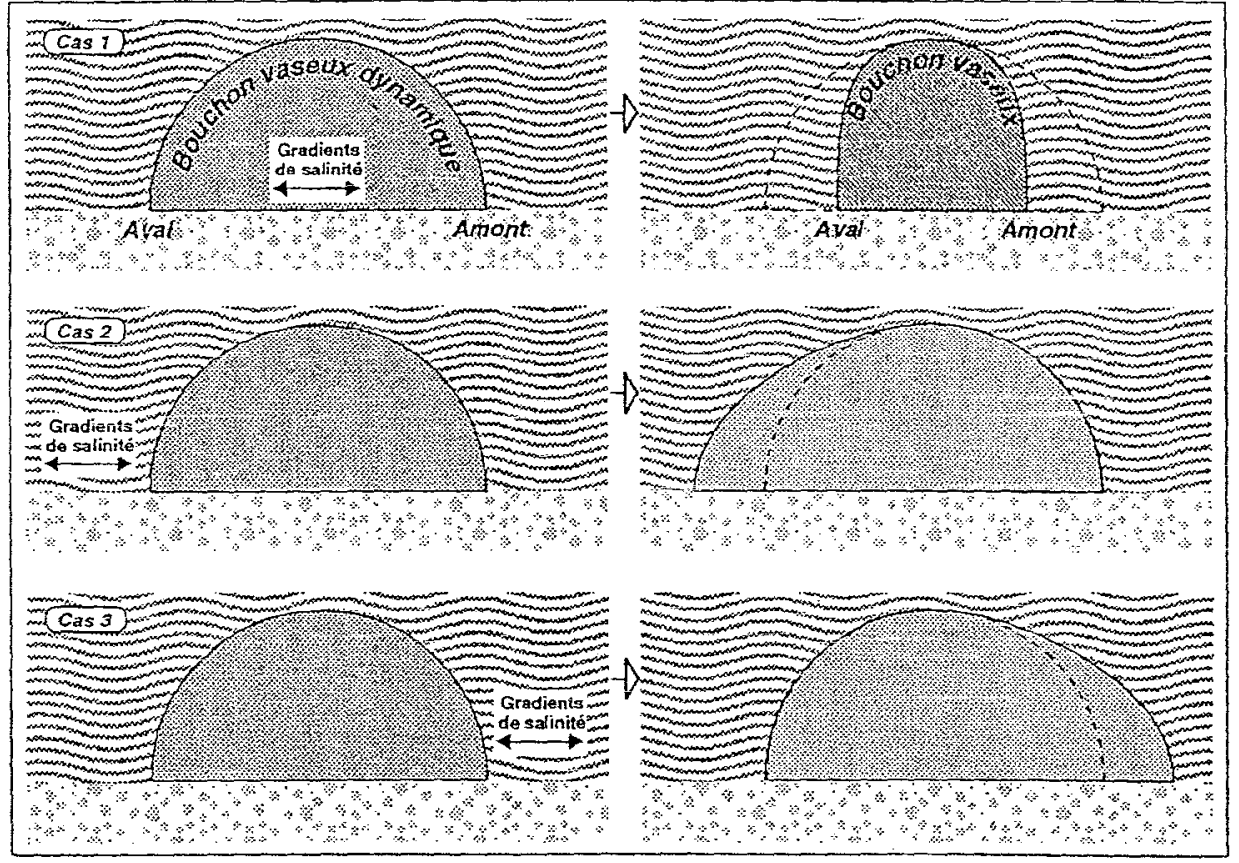

Figure 6: Synthèse de l'influence des gradients de densité sur le comportement des suspensions en vive eau. Colonne de gauche : localisation relative du bouchon vaseux dynamique et des gradients de densité. Colonne de droite : bouchon vaseux résultant de la superposition du bouchon vaseux dynamique et des gradients de densité. 


\section{Conclusion et perspectives}

En utilisant un modèle tridimensionnel, les mécanismes responsables de la formation du bouchon vaseux ont pu être hiérarchisés (figure 7). Les facteurs dynamiques (asymétrie de la propagation de la marée et écoulement fluvial) provoquent un "pompage des sédiments par la marée", qui génère une accumulation d'origine "dynamique". Ces facteurs sont modulés par le comportement du matériel (qui a un rôle important sur la concentration et la localisation des structures turbides) et par la bathymétrie (influence de la localisation des pièges sédimentaires sur les matières en suspension). Les gradients de salinité, dépendants de l'écoulement fluvial, agissent sur les structures turbides par la localisation de la limite de la remontée saline et par l'influence de la circulation de densité induite. Ce phénomène provoque l'apparition d'une accumulation "de densité", indépendante du comportement du matériel. Le bouchon vaseux est le résultat de la superposition, non-linéaire, de ces deux accumulations.

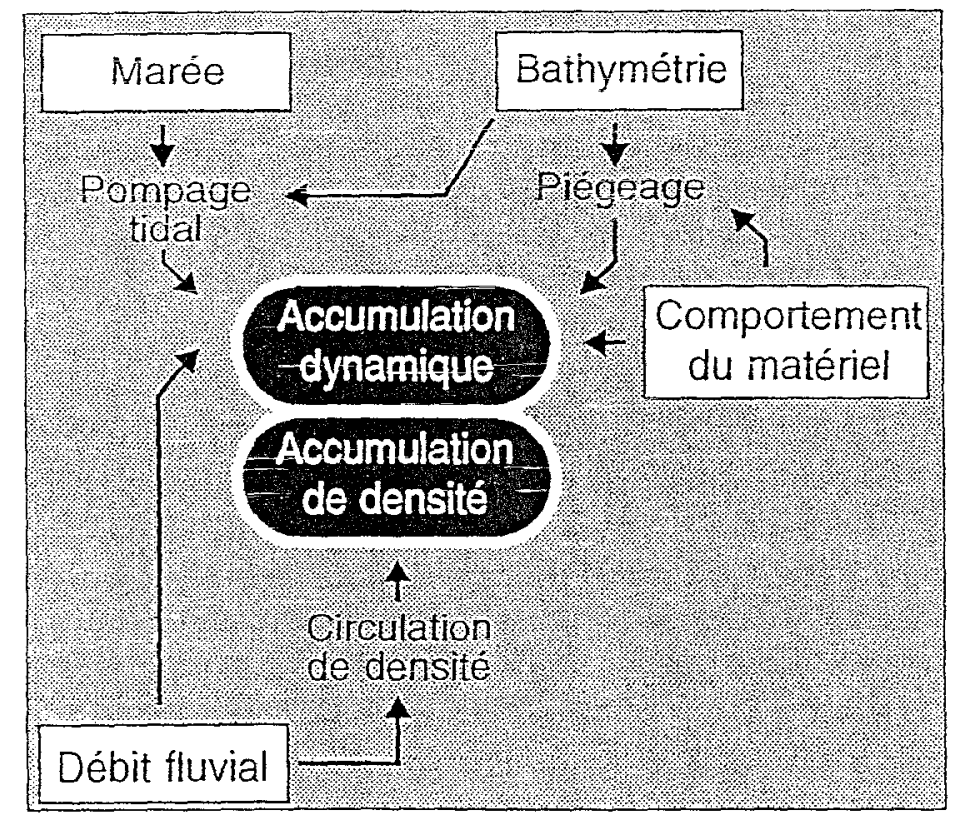

Figure 7 : Hiérarchisation des facteurs responsables de la formation du bouchon vaseux dans l'estuaire de la Seine

Des formulations plus sophistiquées des processus sédimentologiques devraient permettre une simulation plus précise des structures sédimentaires. En particulier, une formulation de la vitesse de chute en fonction de la concentration en sédiments, de la salinité et de la turbulence aurait l'intérêt de permettre la simulation de la floculation et de la défloculation. De même, cette étude pourrait être affinée par la validation des processus de consolidation dans le modèle bidimensionnel horizontal et par leur prise en compte dans le modèle tridimensionnel. Enfin, il faut rappeler l'importance du rôle joué par les piégeages temporaires en morte-eau, qui mériteraient une validation in-situ. 


\section{Remerciements}

Les auteurs remercient vivement B. Simon (SHOM), A. Delouis (Port Autonome de Rouen), P. Bassoullet, P. Cugier et B. Thouvenin (IFREMER) pour leur aide et leurs conseils avisés. Ce travail a été réalisé dans le cadre du programme scientifique Seine Aval, financé par les partenaires suivants : l'Etat, la Région Haute-Normandie et les autres Régions du Bassin Parisien, l'agence de l'eau Seine-Normandie et les industriels de Haute-Normandie.

\section{Bibliographie}

Allen, G.P., Salomon, J.C., Bassoullet, P., Du Penhoat, Y. et De Grandpré, C., 1980. Effects of tides on mixing and suspended sediment transport in macrotidal estuaries. Sedimentological Geology, 26: 69-90.

Avoine J., 1981. L'estuaire de la Seine : sédiments et dynamique sédimentaire. Thèse Université de Caen. $236 \mathrm{p}$.

Avoine, J., Lesueur, P. et Rouault, T., 1996. Evolution morphologique et couverture sédimentaire de l'estuaire de la Seine - Actualisations et fluctuations. Rapport final par laboratoire du programme scientifique Seine Aval. Thème : hydrodynamique et transport sédimentaire. pp 2-20.

Bott A., 1989. A positive definite advection scheme obtained by non-linear renormalization of the advective fluxes. Monthly weather review, vol. 117, pp. 1006-1015.

Brenon, I., 1997. Modélisation de la dynamique des sédiments fins dans l'estuaire de la Seine. Thèse de l'Université de Bretagne Occidentale. 205 p.

Brenon, I. et Le Hir, P., 1996a. Modélisation de la dynamique des sédiments fins dans l'estuaire de la Seine. Actes de colloque des IVèmes Journées Génie Civil - Génie Côtier.

Brenon, I. et Le Hir, P., 1996b. Modelling fine sediment dynamics in the Seine estuary: interaction between turbidity patterns and sediment balance. Proceedings of the $8^{\text {th }}$ International Biennal Conference on Physics of Estuaries and Coatal Seas To be published.

Cugier P., 1997. Modélisation du devenir à moyen terme dans l'eau et le sédiment des éléments majeurs (C,N,P,Si, O) rejetés par la Seine en Baie de Seine. Rapport IFREMER de thèse.

Defossez, J.P., 1996. Dynamique des macrofiocs au cours de cycles tidaux. Mlise au point d'un système d'observation : Vidèo In Lab. Rapport de D.E.A. de l'Université de Rouen. 55 p.

De Nadaillac, G., 1985. Transport en suspension des vases : étude bibliographique. Rapport IFREMER n'DERO-85. $132 \mathrm{p}$.

Krone, R.B., 1962. Flume studies of the transport of sediment in estuarine shoaling processes. Technical Report of Hydraulic Engineering Laboratory. University of California.

Le Hir, P., 1997. Filuid and sediment "integrated" modelling. Application to fluid mud flows in estuaries. Proceedings of INTERCOH'94, Wallingford, 11-15 juillet, Eds Wiley, pp.417-428.

Partheniades, E., 1965. Erosion and deposition of cohesive soils. Journal of the Hydraulics Division, ASCE, Proceedings, Vol. 91, n HY1, pp. 105-139.

Postma, H., 1961. Suspended transport and sedimentation in the estuarine environment. In: G.H. Lauff (Editor), Estuaries. American Association of Advanced Science. Vol. 83. pp 158-179.

Toorman, 1993. A study of erosion and deposition of cohesive sediment with a 1-point transport model. Report n ${ }^{\circ}$ HYD147. MAST G8M Coastal Morphodynamics.

Van Leussen, W.,1995. estuarine macroflocs : their role in fine grained sediment transport. Thèse de 1 Université d'Utrecht. pp. 488. 